Escherichia coli causing primary pyelonephritis. Lancet 1981; ii: 1366-69.

24 Korhonen TK, Valtonen MV, Parkkinen J, et al. Serotype, hemolysin production, and receptor recognition of Escherichia coli stains associated with neonatal sepsis and meningitis. Infect Immun 1985; 48: 486-91.

25 Old DC. Inhibition of the interaction between fimbrial hemagglutinins and erythrocytes by $\mathrm{D}$-mannose and other carbohydrates. $\mathcal{f}$ Gen Microbiol 1972; 71: 149-57.

26 Green CP, Thomas VL. Hemagglutination of human type O erythrocytes, hemolysin production and serogrouping of Escherichia coli isolates from patients with acute pyelonephritis cystitis, and asymptomatic bacteriuria. Infect Immun 1981; 31: 309-15.

27 Okada Y, Tsuji T. Immunohistochemical application of monoclonal antibodies to reveal the structure and localization of carbohydrate antigens: $\mathrm{N}$-acetyl-lactosamine related carbohydrates antigens in human biliary epithelial cells. F Immunol Method 1988; 112: 243-49.

28 Sung JY, Costerton JW, Shaffer EA. Defense system in the biliary tract against bacterial infection. Dig Dis Sci 1992; 37: 689-96.

29 Hamadeh RM, Mandrell RE, Griffiss JM. Immunophysical characterization of human isolates of Serratia marcescens. $\mathcal{F}$ Clin Microbiol 1990; 28: 20-26.

\title{
Risk of endometrial cancer after tamoxifen treatment of breast cancer
}

\author{
Flora E van Leeuwen, Jantien Benraadt, Jan Willem W Coebergh, Lambertus A L M Kiemeney, Charles HF Gimbrère, \\ Renée Otter, Leo J Schouten, Ronald A M Damhuis, Marijke Bontenbal, Fred W Diepenhorst, \\ Alexandra $W$ van den Belt-Dusebout, Harm van Tinteren
}

\section{Summary}

Since large trials have been set up to assess whether tamoxifen decreases the risk of breast cancer in healthy women, it has become important to investigate the drug's potential adverse effects, including occurrence of endometrial cancer. We undertook a case-control study in the Netherlands to assess the effect of tamoxifen on the risk of endometrial cancer after breast cancer.

Through the population-based Netherlands Cancer Registry and two older, hospital-based, registries, we identified 98 patients who had endometrial cancer diagnosed at least 3 months after a diagnosis of primary breast cancer. Detailed information about treatment was obtained for all these patients, and for 285 controls, who were matched to the cases for age, year of breast cancer diagnosis, and survival time with intact uterus. Tamoxifen had been used by $24 \%$ of patients with subsequent endometrial cancer and $20 \%$ of controls (relative risk 1.3 [95\% $\mathrm{Cl} 0.7-2 \cdot 4]$ ). Women who had used tamoxifen for more than 2 years had a 23 ( 0 9-5 9) times greater risk of endometrial cancer than never users. There was a significant trend of increasing risk of endometrial cancer with duration of tamoxifen use $(p=0049)$, and also with cumulative dose $(p=0.046)$. The duration-response trends were similar with daily doses of $40 \mathrm{mg}$ or $30 \mathrm{mg}$ and less.

These findings support the hypothesis that tamoxifen use increases the risk of endometrial cancer. This oestrogenic effect on the endometrium was not related to the dose intensity. Physicians should be aware of the higher risk of endometrial cancer in tamoxifen users

Lancet 1994; 343: 448-52

Department of Epidemlology, Netherlands Cancer Institute, Amsterdam (F E van Leeuwen MSPH, A W van den Belt-Dusebout); Comprehenslve Cancer Centers-Amsterdam ( $F E$ van Leeuwen, J Benraadt MD, FW Diepenhorst, $H$ van Tinteren MSc), South Netherlands ( $J W$ W Coebergh MD), East Netherlands

(L A L M Kiemeney PhD), Middle Netherlands (C H F Gımbrère MD), Northern Netherlands (R Otter MD), Limburg (L J Schouten MD), Rotterdam (R A M Damhuis MD), and Stedendriehoek Twente (J Benraadt); and Dr Danlel den Hoed Cancer Center, Rotterdam (M Bontenbal MD), Netherlands

Correspondence to: Ms Flora E van Leeuwen, Department of Epidemiology, Netherlands Cancer Institute, Plesmanlaan 121, 1066 CX, Amsterdam, Netherlands

\section{Introduction}

Since its introduction in the early 1970s, tamoxifen has been widely and effectively used to treat advanced breast cancer. Adjuvant tamoxifen therapy in early-stage breast cancer became common in the $1980 \mathrm{~s}$, and has been convincingly shown to improve disease-free survival as well as overall survival for women older than 50 years. ${ }^{1}$ Several large trials have suggested, furthermore, that tamoxifen reduces the risk of cancer in the contralateral breast. ${ }^{1-4}$ Based on these findings, chemoprevention trials have been set up in the USA and Europe to find out whether tamoxifen decreases the risk of breast cancer developing in healthy women judged to be at high risk of the disease..$^{5.6}$

Our knowledge of the long-term effects of tamoxifen is still limited. Tamoxifen's mixed oestrogen-agonist and oestrogen-antagonist properties have led to concern that the drug may increase the risk of endometrial cancer. The Stockholm trial ${ }^{4}$ found that women receiving $40 \mathrm{mg}$ tamoxifen daily for at least 2 years had a more than six-fold excess risk of endometrial cancer in comparison with untreated controls. No significant increase in risk has been reported in other major adjuvant trials, ${ }^{7}$ although a non-significant excess of endometrial cancer after tamoxifen treatment was noted in an evaluation of Danish trials and in an unpublished Southwest Oncology Group Trial. ${ }^{8-10}$ The 10-year cumulative risk of endometrial cancer in postmenopausal women is estimated to be $0.3 \%$ or less. Thus, a follow-up study with sufficient power to settle the question of whether there is an excess risk of endometrial cancer after tamoxifen treatment would require long-term follow-up of many thousands of breast cancer patients. We therefore used a case-control design to investigate, in a nationwide study, whether tamoxifen, at different doses and for different durations, increases the risk of endometrial cancer.

\section{Patlents and methods}

Since 1989, the Netherlands has had a population-based, nationwide cancer registry served by nine regional cancer registries. ${ }^{11}$ Most registries have been operating since 1986, the Eindhoven Cancer Registry since 1975, ${ }^{12}$ and the Middle Netherlands Breast Cancer Registry since $1973 .{ }^{13}$ Patients with endometrial cancer after breast cancer were identified from eight of the nine regional cancer registries; other cases (most with endometrial cancer diagnosed before 1986) were identified from the hospital tumour registries of two major cancer treatment 
centres (the Netherlands Cancer Institute [NKI], Amsterdam, and the Dr Daniel den Hoed Cancer Center [DDHK], Rotterdam).

Women were eligible for the study if they had a histologically or cytologically confirmed diagnosis of infiltrating endometrial carcinoma at least 3 months after a diagnosis of primary breast cancer; if the breast cancer developed after Jan 1, 1976 (Jan 1, 1972 in NKI and DDHK; before these dates tamoxifen was nor commonly used); and if they had no other second malignant disorder after breast cancer, except for carcinoma in situ of the uterine cervix, basal-cell carcinoma of skin, or contralateral breast cancer. We required that the diagnosis of breast cancer had been made in a hospital that, at the time, was taking part in any type of registration of all breast cancer cases diagnosed in that hospital. This eligibility criterion was necessary because the method of selecting controls was based on calendar year of breast cancer diagnosis. 98 patients with endometrial cancer after breast cancer met these eligibility criteria.

Controls were women with breast cancer in whom endometrial cancer had not been diagnosed. For each case patient, 3 controls were sought. They were individually matched to the patient for date of birth (within 3 years), date of diagnosis of breast cancer (within 2 years), and pathology laboratory where the breast cancer diagnosis was made. In addition, each control had to have survived, with an intact uterus, for at least as long as the time between the diagnoses of breast cancer and endometrial cancer in the corresponding case. Furthermore, we required that the control had not had a second primary cancer (other than carcinoma in situ of the cervix, basal-cell carcinoma of skin, or contralateral breast cancer) before the date the case patient developed endometrial cancer. When more than 3 controls per case met the above criteria, we selected those with the closest year of diagnosis of breast cancer, and then closest date of birth, to the case. For endometrial cancer patients whose breast cancer diagnoses were recorded in the cancer registries, controls were drawn through the registries. For those whose breast cancer registration records were not present in the registries (since they were not operating at the time), controls were selected through the Dutch Network and National Database for Pathology. This database contains records of all cytological and histological diagnoses made in the Netherlands, with computerised data submission by the individual pathology laboratories. The database was set up in 1977, and complete national coverage was achieved in 1989. Control selection through this database was allowed only if the breast cancer diagnosis of the case patient was also recorded in the pathology database. 3 controls were found for 91 case patients, 2 controls for 5 cases, and 1 control for 2 cases.

For each case and her matched controls, full medical records were obtained for detailed data abstraction. Information was collected on stage of breast cancer, menopausal status at diagnosis of breast cancer, occurrence of contralateral disease, vital status, date of latest follow-up examination or date of death, and cause of death. We recorded for each period of tamoxifen treatment the starting date, the stopping date, and the dosage. The use of radiotherapy, chemotherapy, and hormonal treatment other than tamoxifen for breast cancer was also recorded. For each control, we checked extensively whether she had undergone hysterectomy (in which case she was excluded from the study). If necessary, information was obtained from the patient's general practitioner. Complete information on all treatments, including periods of tamoxifen use and dosage, was eventually available for all women. For cases, we also sought information from the medical record on the endometrial cancer diagnosis (stage of endometrial cancer, full pathology report, treatment for endometrial cancer). All pathology reports of endometrial cancer were reviewed centrally by one of us (JB). If reports were incomplete or diagnosis uncertain, the slides were reviewed by the local pathologist.

The relative risk of endometrial cancer associated with tamoxifen use was estimated by comparison of the case patient's treatment history with that of her matched controls, by conditional logistic regression methods. ${ }^{14}$ Relative risk estimates, two-sided $\mathrm{p}$ values, and $95^{\circ} \circ \mathrm{CI}$ were calculated with the microcomputer programme EGRET (SERC, Seattle, Washington, USA); comparisons between exposure categories were based on likelihood-ratio tests. For analysis, only the treatment of case

\begin{tabular}{|c|c|c|}
\hline & \multicolumn{2}{|l|}{ No $(\%)$} \\
\hline & Cases $(n=98)$ & Controls $(n=285)$ \\
\hline \multicolumn{3}{|l|}{ Age at dlagnosis $(y r)^{*}$} \\
\hline$\leqslant 55$ & $13(13)$ & $36(13)$ \\
\hline $56-65$ & $36(37)$ & $103(36)$ \\
\hline $66-75$ & $31(32)$ & $97(34)$ \\
\hline$>75$ & $18(18)$ & $49(17)$ \\
\hline \multicolumn{3}{|l|}{ Year of dlagnosis* } \\
\hline $1972-1979$ & $26(27)$ & $69(24)$ \\
\hline $1980-1983$ & $21(21)$ & $69(24)$ \\
\hline $1984-1986$ & $22(23)$ & $67(24)$ \\
\hline $1987-1990$ & $29(30)$ & $80(28)$ \\
\hline \multicolumn{3}{|c|}{ The between dlagnoses (mo) $\dagger$} \\
\hline$\leqslant 18$ & $21(21)$ & \\
\hline $19-36$ & $31(32)$ & \\
\hline $37-72$ & $26(27)$ & \\
\hline$>72$ & $20(20)$ & \\
\hline \multicolumn{3}{|l|}{ Stage of breast cancer } \\
\hline Localised & $51(52)$ & $152(53)$ \\
\hline Regional & $36(37)$ & $122(43)$ \\
\hline Distant & $9(9)$ & $10(4)$ \\
\hline Unknown & $2(2)$ & 1 \\
\hline \multicolumn{3}{|c|}{ Menopausal status at dlagnosis* $\ddagger$} \\
\hline Premenopausal & $6(6)$ & $28(10)$ \\
\hline Postmenopausal & $90(92)$ & $254(89)$ \\
\hline Perimenopausal & $2(2)$ & 1 \\
\hline Unknown & 0 & 2 \\
\hline \multicolumn{3}{|l|}{ Treatment\$ } \\
\hline Radiotherapy & $65(66)$ & $212(74)$ \\
\hline Chemotherapy & $7(7)$ & $22(8)$ \\
\hline Tamoxifen treatment & $23(24)$ & $58(20)$ \\
\hline Other hormonal therapy & $6(6)$ & $16(6)$ \\
\hline
\end{tabular}

*Breast cancer $\uparrow$ Breast and endometrial cancers. $\$$ Premenopausal = last menstruation

$<3$ mo previously; postmenopausal = last menstruation $>1 \mathrm{yr}$ previously. STreatment within matched intervals; percentages add to more than 100 because of overlapping categories.

Table 1: Characteristics of case patients with endometrial cancer after breast cancer and matched controls

patients during the time between the diagnoses of breast cancer and of endometrial cancer was included. For controls, treatment was studied for an equivalent time, starting with the date that breast cancer was diagnosed. Total duration of tamoxifen use and total dose were examined specifically as relevant factors in determining risk. The cumulative dose of tamoxifen was grouped into quartiles to calculate relative risks for each category in relation to the reference group of patients not treated with tamoxifen. Tests for trend in relative risk of endometrial cancer by duration (or cumulative dose) of tamoxifen were calculated by fitting the actual months (or $\mathrm{mg}$ ) of use as a continuous variable in the logistic regression analyses. To assess the effect of dose intensity, duration-response slopes were estimated simultaneously for women who had received daily doses of $40 \mathrm{mg}$ and $30 \mathrm{mg}$ or less. Multivariate analyses were done to account for potentially confounding effects of radiotherapy (yes/no), chemotherapy (yes/no), hormonal treatment other than tamoxifen, and breast cancer stage. For this purpose, hormonal treatment other than tamoxifen was classified according to the expected effect on the endometrium (oestrogen stimulation, yes/no; progestagenic protection yes/no).

\section{Results}

Most of the women were over 55 years old and postmenopausal when breast cancer was diagnosed (table 1). Date of birth was matched within 1 year for $78 \%$ of controls and within 2 years for $94 \%$. Most $(74 \%)$ of the endometrial cancers occurred in patients whose breast cancer had been diagnosed in the $1980 \mathrm{~s} .89 \%$ of controls matched cases within 1 year for diagnosis of breast cancer. The median time between the diagnoses of breast cancer and endometrial cancer was 34 (5-201) months. More cases than controls were diagnosed with advanced breast cancer ( 9 vs $4 \%, p=0.03$, Fisher's exact test). Controls were slightly more likely than case patients to have received 


\begin{tabular}{|c|c|c|c|}
\hline & Cases & Controls & $\begin{array}{l}\text { Matched } \\
\text { RR (95\% CI) }\end{array}$ \\
\hline \multicolumn{4}{|l|}{ Duration (mo) } \\
\hline Never & 75 & 227 & 10 \\
\hline$\leqslant 12$ & 6 & 28 & $06(02-17)$ \\
\hline $13-24$ & 7 & 14 & $19(06-58)$ \\
\hline $25-60$ & 7 & 12 & $22(08-65)$ \\
\hline$>60$ & 3 & 4 & $30(06-158)$ \\
\hline \multicolumn{4}{|c|}{ Cumulative dose (mg) } \\
\hline 0 & 75 & 227 & 10 \\
\hline $1-8670$ & 3 & 17 & $07(02-23)$ \\
\hline $8671-14680$ & 5 & 16 & $08\left(\begin{array}{ll}0 & 2-2 \\
6\end{array}\right)$ \\
\hline $14681-31720$ & 8 & 12 & $25(09-71)$ \\
\hline$>31720$ & 7 & 13 & $21\left(\begin{array}{lll}07-60) & 0\end{array}\right.$ \\
\hline
\end{tabular}

Table 2: Relative risk (RR) of endometrlal cancer according to total duration of tamoxifen use and cumulative dose

radiotherapy for breast cancer. However, there were no significant differences between endometrial cancer patients and controls in the types of treatments used for breast cancer.

$24 \%$ of the case patients and $20 \%$ of controls had used tamoxifen at some time, and the relative risk of endometrial cancer for women who had ever been treated with tamoxifen compared with those who had not was $1.3(95 \%$ CI $0 \cdot 7-2 \cdot 4)$. The median duration of tamoxifen use was higher for case patients than for controls (19 vs 13 months, $p=0.07)$. The risk of endometrial cancer increased with duration (table 2). Women who had used tamoxifen for more than 2 years had a $2 \cdot 3(0.9-5 \cdot 9)$ times greater risk of endometrial cancer than never users and the risk rose to $3 \cdot 0$ $(0 \cdot 6-15 \cdot 8)$ for those treated for more than 5 years. None of the risk estimates for categories of duration reached significance, but the inclusion of duration of tamoxifen use as a linear continuous variable in the logistic regression model produced a significant trend $(p=0 \cdot 049)$. The cumulative dose of tamoxifen was also associated with the risk of endometrial cancer (table $2, p$ for trend $=0.046$ ). The effect of tamoxifen was best fitted by including a binary term (ever/never use) and the log-transformed duration of tamoxifen use as a continuous variable in the model

\begin{tabular}{|c|c|c|}
\hline & No $(\%)$ & \\
\hline & Never users $(n=75)$ & Ever users $(n=23)$ \\
\hline \multicolumn{3}{|l|}{ Stage* } \\
\hline 1 & $62(83)$ & $17(74)$ \\
\hline ॥ & $7(9)$ & $3(13)$ \\
\hline III & $1(1)$ & 0 \\
\hline IV & $1(1)$ & 0 \\
\hline Unknown & $4(5)$ & $3(13)$ \\
\hline \multicolumn{3}{|l|}{ Morphology } \\
\hline Adenocarcinoma & $54(72)$ & $15(65)$ \\
\hline Papıllary adenocarcinoma & $13(17)$ & $4(17)$ \\
\hline Clear cell adenocarcinoma & $2(3)$ & $1(4)$ \\
\hline Adenosquamous carcinoma & $3(4)$ & $1(4)$ \\
\hline Adenoacanthoma & $3(4)$ & $2(9)$ \\
\hline \multicolumn{3}{|l|}{ Treatment } \\
\hline Surgery alone & $29(39)$ & $11(48)$ \\
\hline Surgery+RT & $34(45)$ & $6(26)$ \\
\hline Surgery $+\mathrm{HT}$ & $6(8)$ & $4(17)$ \\
\hline$H T \pm R T$ & $2(3)$ & $1(4)$ \\
\hline None & $4(5)$ & $1(4)$ \\
\hline \multicolumn{3}{|l|}{ Deaths } \\
\hline Total & $25(33)$ & $9(39)$ \\
\hline Breast cancer & 13 & 4 \\
\hline Endometrial cancer & 4 & 0 \\
\hline All other causes & 5 & 4 \\
\hline Unknown & 3 & 1 \\
\hline
\end{tabular}

$\mathrm{RT}=$ radiotherapy; $\mathrm{HT}=$ hormonal therapy. ${ }^{*} \mathrm{FIGO} .^{16}$

Table 3: Characteristics of patients with endometrial cancer according to use of tamoxifen $(p=0.026)$. Similar duration and dose-response patterns were found when analyses were restricted to the 89 women (and their matched controls) in whom endometrial cancer was diagnosed more than 1 year after the initial breast cancer diagnosis. Adjustment for the variables chemotherapy, radiotherapy, hormonal treatment other than tamoxifen, and stage of breast cancer did not substantially alter the risk estimates in table 2 .

Of the 81 tamoxifen users in our study, most $(59 \%)$ had received daily doses of $40 \mathrm{mg}, 17 \%$ received $30 \mathrm{mg}$, and $23 \%$ received $20 \mathrm{mg}$ or less. We attempted to separate the effects of dose intensity, duration of tamoxifen use, and cumulative dose. The average dose of tamoxifen taken daily did not affect the risk of endometrial cancer in a model accounting for total duration of use $(p=0.54)$. The inclusion of dose intensity in the model hardly affected the duration-response trend $(0.05)$. To explore further the effect of dose intensity, duration-response slopes were fitted simultaneously for women who had received $40 \mathrm{mg}$ daily and those who received $30 \mathrm{mg}$ or less. The relative risk per year of tamoxifen use (with a multiplicative model, based on actual months of use) was 1.22 for a daily dose of $30 \mathrm{mg}$ or less and 1.24 for $40 \mathrm{mg}$ (tests for linear trend $p=0 \cdot 18$ and $p=0 \cdot 10$, respectively). The two doses did not differ in duration-response trends $(p=0.73)$.

Within the case group, we studied whether the endometrial cancers in women who had used tamoxifen had different characteristics from those diagnosed in women who had never been treated with the drug (table 3 ). The stage distributions ${ }^{15}$ and morphology of the endometrial cancers in these two groups showed no striking differences. During median follow-up of 15 months after the diagnosis of endometrial cancer, none of the tamoxifen-treated women died of this cancer. Median follow-up in never users was 29 months. 2-year actuarial survival after the diagnosis of endometrial cancer was similar for women who had received breast cancer treatment with tamoxifen and for those who had never received the drug $(68 \%$ and $71 \%$, respectively).

\section{Discusslon}

Our results support the hypothesis that use of tamoxifen increases the risk of endometrial cancer. We found a significant trend in risk of endometrial cancer with total duration of tamoxifen treatment, whatever the dose intensity.

In interpreting our results we considered several potential sources of bias. Increased medical surveillance in tamoxifen users, as well as the presence of gynaecological symptoms due to tamoxifen treatment (eg, vaginal bleeding) might lead to earlier diagnosis of endometrial cancer in such women than in untreated patients, which might result in a spurious association between tamoxifen and endometrial cancer. We found no evidence for such bias. First, the stage distribution of the endometrial cancers did not differ between ever and never users. Furthermore, women who had used tamoxifen for a year or less did not have an increased risk of endometrial cancer, and in the (small) subgroup of endometrial cancers diagnosed within 1 year from the breast cancer diagnosis we found no association with tamoxifen use.

Since the Netherlands did not have a nationwide cancer registry in the 1970s and early 1980s, some patients with endometrial cancer after breast cancer were not eligible for our study, solely because there was no sampling frame for control breast cancer patients diagnosed in the same 
calendar period. Although this feature reduces the study's power, we do not believe that it introduces bias to our results. For each endometrial cancer case included in the study, all the corresponding matched controls were drawn from a source that also included the breast cancer diagnosis of the case patient. Given this design, selection bias could arise only in the unlikely event that an association between tamoxifen and risk of endometrial cancer depended on the presence of a regional cancer registry at the time of the breast cancer diagnosis.

By matching controls with case patients, we incorporated adjustment for important confounders such as age and calendar year at breast cancer diagnosis. The additional matching, according to the pathology laboratory where the breast cancer diagnosis was made, was introduced for practical reasons, and enabled us to include case patients with a long time between the diagnoses of endometrial and breast cancer. However, matching for pathology laboratory may have resulted in greater similarity in treatment between case patients and controls than would otherwise have been observed. This notion is not just theoretical, since the introduction date of tamoxifen, the indications for its use, and the dose and duration of administration have varied between regions in the Netherlands. Overmatching may thus have led us to underestimate risk of endometrial cancer associated with tamoxifen use. On the other hand, we may have adjusted for determinants of regional differences in incidence of endometrial cancer. Since the possibility of overmatching cannot be excluded, our risks must be viewed as minimum estimates of the true effect of tamoxifen.

Evidence for an association between the use of tamoxifen and risk of endometrial cancer has, to date, come from only two other studies. In the Stockholm trial ${ }^{4}$ the relative risk was $6.4(p<0.01)$ for women who received $40 \mathrm{mg} /$ day tamoxifen for at least 2 years compared with untreated women. In the Danish studies ${ }^{8,9}$ there was a non-significant three-fold excess in women who received $30 \mathrm{mg}$ daily for 48 weeks compared with untreated women. These estimates were based on 15 and 20 cases of endometrial cancer, respectively. Our risk estimates, based on much larger numbers of cases, are somewhat lower than these previous estimates (relative risk 2.3 for 2 years' use, 3.0 for $>5$ years' use), possibly because of overmatching in the study design. In several other large trials of adjuvant tamoxifen therapy, no increased risk of endometrial cancer has been observed..$^{3,716}$ However, no full statistical evaluation of second cancer incidence in these trials has been published, and incomplete registration of second cancers, or lack of sufficient follow-up time, may have led to negative findings in some trials.

The observation of increased risk of endometrial cancer after tamoxifen treatment is biologically plausible and may be explained as a partial oestrogen-agonistic effect of tamoxifen on the endometrium. ${ }^{17,18}$ Endometrial lesions, such as hyperplasia, are more common in breast cancer patients who have received a cumulative dose of tamoxifen of $15 \mathrm{~g}$ or more than in those receiving smaller doses. ${ }^{19}$ Interestingly, in our study the risk of endometrial cancer was also increased only after cumulative doses above $15 \mathrm{~g}$. It has been suggested that the oestrogenic effect of tamoxifen, especially the proliferative effect on the endometrium, depends upon the dose intensity of the drug. Our results do not support this suggestion. We must emphasise, however, that few women in our study received $30 \mathrm{mg}$ daily or less, and neither of the duration-response trends for the two subcategories of tamoxifen dose intensity reached statistical significance. Numbers did not permit a separate risk calculation for women who took $20 \mathrm{mg}$ per day, which is the dose used in the continuing chemoprevention trials. The Danish study ${ }^{8,9}$ also reported increased risk (though not significantly) of endometrial cancer at a dose of $30 \mathrm{mg}$. Oestrogenic effects of a daily dose of $20 \mathrm{mg}$ tamoxifen on the postmenopausal vagina have also been reported..$^{20,21} \mathrm{With}$ regard to a similar association, that between postmenopausal oestrogen replacement therapy and risk of endometrial cancer, elevated risks have also been reported for the lowest dose levels. ${ }^{22}$

It has been postulated that any increase of endometrial cancer risk due to the oestrogenic effects of tamoxifen would yield a high proportion of highly differentiated, prognostically favourable tumours, as has been observed with endometrial carcinomas associated with oestrogen replacement therapy. ${ }^{23-25}$ We found no substantial differences in histological features of the endometrial cancers diagnosed in tamoxifen-treated patients and in non-treated women. The only suggestion of a difference was in the proportion of well-differentiated tumours $(52 \%$ in tamoxifen users and $32 \%$ in women not treated with tamoxifen). Since the histological slides of endometrial cancers were not reviewed centrally, this finding must be interpreted cautiously. In Magriples and colleagues' study $^{26}$ of the clinical and histological features of endometrial cancers in breast cancer patients who had or had not received tamoxifen, there was a significant excess of poorly differentiated tumours in the tamoxifen-treated group ${ }^{26}$ However, the number of tamoxifen users in that study, as well as in ours, was small (15 and 23, respectively), and in both studies the age distribution of the tamoxifentreated group differed from that in the untreated patients. A much larger patient population would be needed to refute or confirm an association between tamoxifen use and specific histological characteristics of endometrial cancer.

Increased risk of endometrial cancer after tamoxifen use will cause some morbidity in breast cancer patients treated with the drug. However, the proven clinical benefit of tamoxifen in controlling breast cancer ${ }^{1}$ clearly outweighs the modest increase of endometrial cancer risk. Endometrial cancer has a more favourable prognosis than breast cancer, so no patient should be denied tamoxifen treatment of her breast tumour because of anticipated adverse effects on the endometrium.

The issue of whether tamoxifen should be used to prevent cancer in healthy women, who do not have a medical need for treatment, is different, however. Even if, at the population level, the postulated reduction in the incidence of breast cancer were to outweigh the apparently increased risk of endometrial cancer, it is debatable whether the use of a medical intervention can be justified when it prevents breast cancer in some women at the cost of inducing endometrial cancer in others. This issue will continue to provoke discussion in the medical community, ${ }^{27-29}$ until more is known about tamoxifen's long-term benefits and adverse effects. Further studies are needed to quantify the risk for women taking $30 \mathrm{mg}$ or $20 \mathrm{mg}$ per day, to assess the effect of very long durations of use (5 years or more), and to determine the risk for ex-users.

Meanwhile, we believe that, on the basis of our results, physicians should be alert to the higher risk of endometrial cancer in all women using tamoxifen, both breast cancer patients and healthy participants in prevention trials. Regular gynaecological examinations may be worth while for long-term users. 
We thank Dr I J A M G Casparie-van Velsen (Dutch Network and National Database for Pathology), Prof H J A Collette (Department of Epidemiology, University of Utrecht), and the hospital cancer registries of the Netherlands Cancer Institute and the Dr Daniel den Hoed Cancer Center for making control selection possible; the treating specialists for providing access to patients' medical charts; and the abstracting teams from the regional cancer registries for data collection. This study was supported partly by a grant (NKI 88-11) from the Dutch Cancer Society.

\section{References}

1 Early Breast Cancer Trialists' Collaborative Group. Systemic treatment of early breast cancer by hormonal, cytotoxic, or immune therapy: 133 randomised trials involving 31000 recurrences and 24000 deaths among 75000 women. Lancet $1992 ; 339$ : 1-15, 71-85.

2 CRC Adjuvant Breast Trial Working Party. Cyclophosphamide and tamoxifen as adjuvant therapies in the management of breast cancer. Br F Cancer 1988; 57: 604-07.

3 Fisher B, Constantino J, Redmond C, et al. A randomized trial evaluating tamoxifen in the treatment of patients with node-negative breast cancer who have estrogen-receptor-positive tumors. $N$ Engl $\mathcal{f}$ Med 1989; 320: 489-84.

4 Fornander T, Rutqvist LE, Cedermark B, et al. Adjuvant tamoxifen in early breast cancers: occurrence of new primary cancers. Lancet 1989; i: 117-20.

5 Powles TJ, Tillyer CR, Jones AL, et al. Prevention of breast cancer with tamoxifen: an update on the Royal Marsden pilot programme. Eur f Cancer 1990; 26: 680-84.

6 Fisher B, Redmond C. New perspective on cancer of the contralateral breast: a marker for assessing tamoxifen as a preventive agent. $\mathcal{F}$ Natl Cancer Inst 1991; 83: 1278-80.

7 Nayfield SG, Karp JE, Ford LG, Dorr FA, Kramer BS. Potential role of tamoxifen in prevention of breast cancer. $\mathcal{F}$ Natl Cancer Inst 1991 ; 83: $1450-59$.

8 Andersson $\mathrm{M}$, Storm $\mathrm{HH}$, Mouridsen HT. Incidence of new primary cancers after adjuvant tamoxifen therapy and radiotherapy for early breast cancer. $\mathcal{F}$ Natl Cancer Inst 1991; 83: 1013-17.

9 Andersson M, Storm HH, Mouridsen HT. Carcinogenic effects of adjuvant tamoxifen treatment and radiotherapy for early breast cancer. Acta Oncol 1992; 31: 259-63.

10 Sunderland MC, Osborne CK. Tamoxifen in premenopausal patients with metastatic breast cancer: a review. F Clin Oncol 1991; 9: 1283-97.

11 LOK/Coordinating Council of Comprehensive Cancer Centers, ed. Incidence of cancer in the Netherlands 1989: First report of the Netherlands Cancer Registry. Utrecht: LOK, 1992.
12 Muir C, Waterhouse J, Mack T, et al, eds. Cancer incidence in five continents, vol V, no 88. Lyon: IARC Scientific Publications, 1987.

13 Collette HJA, Day NE, Rombach JJ, De Waard F. Evaluation of screening for breast cancer in a non-randomised study (the DOM project) by means of a case-control study. Lancet 1984; i: 1224-26.

14 Breslow NE, Day NE. Statistical methods in cancer research: the analysis of case-control studies, vol 1, no 32. Lyon: IARC Scientific Publications, 1980.

15 Creasman WT. New gynecologic cancer staging. Obstet Gynecol 1990; 75: $287-88$.

16 Stewart HJ, Knight GM. Tamoxifen and the uterus and endometrium Lancet $1989 ; 1: 375-76$

17 Gottardis MM, Robinson SP, Satyaswaroop PS, Jordan VC. Contrasting actions of tamoxifen on endometrial and breast cancer tumor growth in the athymic mouse. Cancer Res 1988; 48: 812-15.

18 Boccardo F, Guarnieri D, Rubagotti A, et al. Endocrine effects of tamoxifen in postmenopausal breast cancer patients. Tumori $1984 ; 70$ 61-68.

19 De Muylder X, Neven P, De Somer M, Van Belle Y, Vanderick G, De Muylder E. Endometrial lesions in patients undergoing tamoxifen therapy. Int f Gynaecol Obstet 1991; 36: 127-30.

20 Ferrazzi E, Cartei G, Mattarazzo R, Fiorentino M. Oestrogen-like effect of tamoxifen on vaginal epithelium. $B M \mathcal{F}$ 1977; i: 1351-52.

21 Boccardo F, Bruzzi P, Rugagotti A, et al. Estrogen-like action of tamoxifen on vaginal epithelium in breast cancer patients. Oncology $1981 ; 38: 281-85$

22 Grady D, Rubin SM, Petitti DB, et al. Hormone therapy to preven disease and prolong life in postmenopausal women. Ann Intern Med 1992; 117: 1016-37.

23 Elwood JM, Boyes DA. Clinical and pathological features and survival of endometrial cancer patients in relation to prior use of estrogens. Gynecol Oncol 1980; 10: 173-87.

24 Buring JE, Bain CJ, Ehrmann RL. Conjugated estrogen use and risk of cancer registries. Am 7 Epidemiol 1986; 124: 434-41.

25 Collins J, Donner A, Allen LN, Adams O. Oestrogen use and survival in endometrial cancer. Lancet 1980; i: 961-14.

26 Magriples U, Naftolin F, Schwartz PE, Carcangiu ML. High-grade endometrial carcinoma in tamoxifen-treated breast cancer patients. 7 Clin Oncol 1993; 11: 485-90.

27 Fugh-Berman A, Epstein S. Tamoxifen: disease prevention or disease substitution? Lancet 1992; 340: 1143-45.

28 Powles TJ. The case for clinical trials of tamoxifen for prevention of breast cancer. Lancet 1992; 340: 1145-47.

29 Neven P. Tamoxifen and endometrial lesions. Lancet 1993; 342: 452.

\section{$\alpha_{1}$-antitrypsin deficiency in intracranial aneurysms and cervical artery dissection}

\author{
Wouter I Schievink, Udaya B S Prakash, \\ David G Piepgras, Bahram Mokri
}

The pathogenesis of ruptured intracranial aneurysms and cervical artery dissections is poorly understood but may be similar in these two disorders. We report four patients with $\alpha_{1}$-antitrypsin deficiency who developed a ruptured intracranial aneurysm or spontaneous dissection of the cervical internal carotid artery. Three patients were heterozygous for the deficient allele (PiMZ or PiGZ) and one was homozygous (PiZZ). A deficiency of $\alpha_{1}$-antitrypsin or one of the other protease inhibitors could result in degradation of the arterial wall through an imbalance between proteolytic enzymes and their inhibitors, thereby predisposing the arterial wall to dissection or aneurysm formation.

Lancet 1994; 343: 452-53
The pathogenesis of ruptured intracranial aneurysms and spontaneous cervical artery dissections is not well understood but an underlying arteriopathy is usually suspected. Except for some patients with fibromuscular dysplasia or certain heritable connective tissue disorders, investigations into the nature of this arteriopathy have been unsuccessful. ${ }^{1}$ Deficiencies of $\alpha_{1}$-antitrypsin or other inhibitors of proteolytic enzymes may have a role in the development of abdominal arterial aneurysms. ${ }^{2,3}$ Such deficiency could undermine the integrity of the vascular extracellular matrix, predisposing the arterial wall to dissection or aneurysm formation. We report a group of patients with $\alpha_{1}$-antitrypsin deficiency who developed aneurysmal subarachnoid haemorrhage or spontaneous cervical artery dissection.

All patients with $\alpha$-antitrypsin deficiency, who were evaluated at the Mayo Clinic between 1976 and 1992, were identified through a computerised and coded diagnostic index. This group consisted of 168 women and 194 men (mean age $48 \cdot 1$ years). All had symptoms of $\alpha_{1}$-antitrypsin deficiency, which had been diagnosed with similar methods throughout the study period. During this time, about 140 patients with spontaneous carotid dissection and 1250 patients with aneurysmal subarachnoid haemorrhage were seen at our institution. 